\title{
Palladium-Catalyzed Regioselective Direct Arylation of Benzofurazans at C4 Position
}

\author{
Imane Idris, ${ }^{a, b}$ Fazia Derridj, ${ }^{a, b}$ Jean-François Soulé, ${ }^{a *}$ and Henri Doucet ${ }^{\text {a* }}$ \\ a Institut des Sciences Chimiques de Rennes, UMR 6226 CNRS-Université de Rennes 1 "Organomet́talliques, Mateŕiaux \\ et Catalyse”, Campus de Beaulieu, 35042 Rennes, France. Fax: (+33)-(0)2-2323-6939, jean-framcois.soule@univ- \\ rennes1.fr and henri.doucet@univ-rennes1.fr \\ b Laboratoire de physique et chimie des Matériaux (LPCM), UMMTO University, BP 17 RP, 15000 Tizi-Ouzou, Algeria.
}

Abstract. The palladium-catalyzed direct arylation of Palladium-catalyzed one-pot C4,C7-diarylation of benzofurazans with aryl bromides to access 4- benzofurazane was also described using a larger amount of arylbenzofurazans proceeds in moderate-to-high yields using aryl bromide. Moreover, the derivatization of 4phosphine-free palladium acetate as the catalyst and potassium acetate as an inexpensive base. A wide variety of (hetero)aryl bromides, including bromopyridine and bromothiophene derivatives has been successfully employed. arylbenzofurazans into 4-arylquinaxolines is also reported.

Keywords: Benzofurazans; $\mathrm{C}-\mathrm{H}$ activation; Catalysis; Palladium; Quinaxolines.

\section{Introduction}

Benzofurazan is an important unit, which found applications in materials sciences. Especially C4 or/and C7 (hetero)aryl benzofurazans are involved in the molecular design of photovoltaic materials (Figure 1).$^{[1]} \quad$ Some 4-arylbenzofurazans also displayed important biological activities such as I, which inhibits the PAS-B domain of the hypoxia inducible factor $2 \alpha ;{ }^{[2]}$ or the benzo $[c][1,2,5]$ oxadiazole-1-oxide II, which has been synthetized for its antimicrobial properties. ${ }^{[3]}$ Recently, electron donor-acceptor type organic semiconductors were designed based on 4heteroarylbenzofurazan (Th-BO-Th) for application as organo-photocatalysts (Figure 1). ${ }^{[4]}$
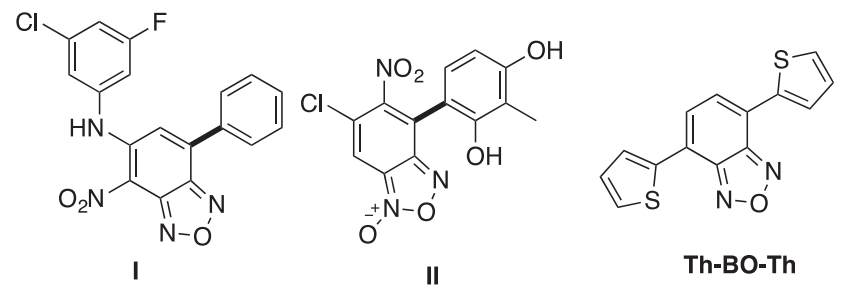

Figure 1. Examples of Useful Molecules Containing a 4Arylbenzofurazan Unit.
One of the most common access to 4arylbenzofurazan derivatives is through a bromination of benzofurazan followed by palladium catalyzed Suzuki ${ }^{[5]}$ or Stille ${ }^{[6]}$ cross-coupling reactions (Scheme 1a). Oligomers have also been synthetized from 4,7-dibromofurazan and an organometallic reagent. ${ }^{[7]}$ Negishi reactions with an organozinc reagent prepared from benzofurazan through a magnesiation with bis(2,2,6,6tetramethylpiperidin-1-yl)magnesium-lithium

chloride complex $\left(\mathrm{TMP}_{2} \mathrm{Mg} \cdot 2 \mathrm{LiCl}\right)$ followed by transmetalation with zinc chloride, have also been reported (Scheme 1b). ${ }^{[8]}$ Since the discovery by Nakamura ${ }^{[9]}$ and Otha ${ }^{[10]}$ of the Pd-catalyzed $\mathrm{C}-\mathrm{H}$ bond arylation of heteroarenes using aryl halides, this technology has emerged as one of the most powerful method for the formation of $\mathrm{C}-\mathrm{C}$ bonds for the access to a wide variety of arylated heterocycles as no prefunctionalization is required. ${ }^{[11]}$ However, examples of palladium-catalyzed non-directed $\mathrm{C}-\mathrm{H}$ bond arylation of 6-membered rings remains scarce. ${ }^{[12]}$ Recent examples on the arylation of activated benzothiadiazole via $\mathrm{C}-\mathrm{H}$ bond activation have been reported using palladium catalysis. ${ }^{[13]}$ To the best of our knowledge there is no example of direct arylation of benzofurazan in the literature, to date (Scheme 1c). 
a. 4-Arylbenzofurazan Synthesis through Suzuki-Miyaura or Stille Couplings $^{5-7}$<smiles>[R]c1cccc2nonc12</smiles>

b. 4-Arylbenzofurazan Synthesis through Negishi Coupling ${ }^{8}$

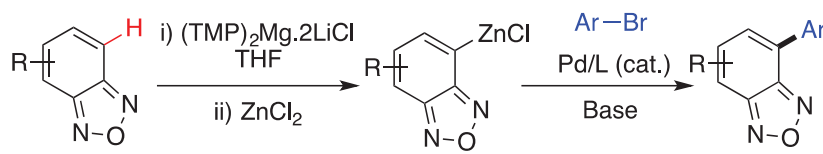

c. Palladium-Catalyzed Direct Arylation of Benzofurazan (this work)

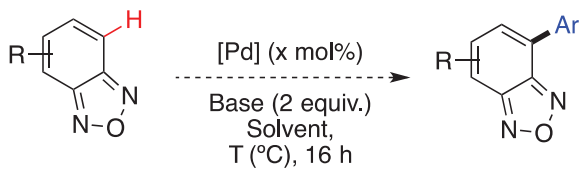

Scheme 1. Previous Strategies to Synthetize 4Arylbenzofurazans.

As part of our continuing efforts concerning the functionalization of benzene rings fused to heterocycles via palladium-catalyzed $\mathrm{C}-\mathrm{H}$ bond arylation, we recently reported an efficient protocol for the synthesis of benzothiazoles and benzoxazoles arylated at the $\mathrm{C} 7$ position. ${ }^{[14]}$ Based on these results, we examined the reactivity of benzofurazan in palladium-catalyzed direct arylation using aryl bromides as aryl source.

\section{Results and Discussion}

We began our investigations by fine-tuning the conditions using benzofurazan and 4bromobenzonitrile as model substrates in 1.5:1 ratio (Table 1). We were pleased to find that the reaction occurred regioselectively at the $\mathrm{C} 4$ position to afford 1 in good yields using $2 \mathrm{~mol} \%$ of a diphosphinepalladium catalyst in the presence of KOAc as base in DMA at $150{ }^{\circ} \mathrm{C}$ or even at $120^{\circ} \mathrm{C}$ (Table 1 , entries 1 and 2). More interestingly, we found that the reaction is also operative using $2 \mathrm{~mol} \%$ of phosphine-free $\mathrm{Pd}(\mathrm{OAc})_{2}$ catalyst in a comparable yield (Table 1 , entry 3). A lower catalyst loading of $1 \mathrm{~mol} \%$ of $\mathrm{Pd}(\mathrm{OAc})_{2}$ gave 1 in $85 \%$ yield; while partial conversion was obtained if only $0.5 \mathrm{~mol} \%$ catalyst loading was used (Table 1, entries 4 and 5). Other inorganic bases, such as PivOK, $\mathrm{K}_{2} \mathrm{CO}_{3}$ or $\mathrm{Cs}_{2} \mathrm{CO}_{3}$ did not allow to improve the yield in $\mathbf{1}$ (Table 1, entries 6-8). When the reaction was performed using $1 \mathrm{~mol} \% \mathrm{Pd}(\mathrm{OAc})_{2}$ at $120{ }^{\circ} \mathrm{C}$, the $\mathrm{C} 4$-arylated benzofurazan 1 was obtained in $87 \%$ yield (Table 1 , entry 9). Under these conditions, the excess of benzofurazan can be decreased up to 1.15 equivalents, as the desired product $\mathbf{1}$ was isolated in $89 \%$ yield (Table 1, entry 10). Then, we employed greener solvents than DMA such as cyclopentylmethyl ether, 1-pentanol or diethylcarbonate, ${ }^{[15]}$ but no reaction occurred (Table 1, entries 11-13). No reaction occurred when 4-chlorobenzonitrile was used as aryl source (Table 1, entry 14). It should also be mentioned that using the optimized reaction conditions, benzothiadiazole was unreactive.

Table 1. Optimization of the Reaction Conditions

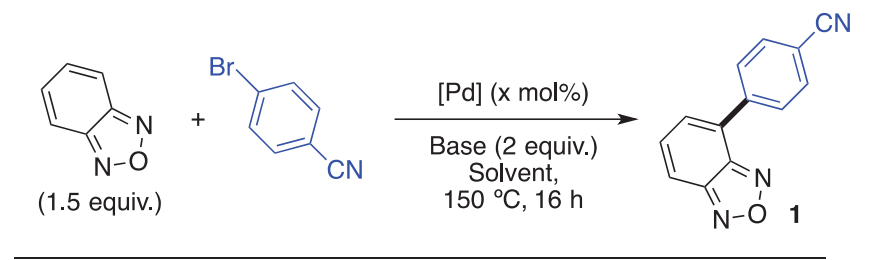

\begin{tabular}{|c|c|c|c|c|c|c|}
\hline Entry & $\begin{array}{c}\mathrm{Pd} \\
(\mathrm{x} \mathrm{mol} \%) \\
\end{array}$ & Base & $\begin{array}{c}\text { Solve } \\
\text { nt }\end{array}$ & $\begin{array}{l}\text { Conv. } \\
(\%)^{[a]}\end{array}$ & $\begin{array}{c}\text { Yield } \\
\text { in } 1(\%)^{[\mathrm{a}}\end{array}$ & \\
\hline 1 & $\begin{array}{c}\mathrm{PdCl}\left(\mathrm{C}_{3} \mathrm{H}_{5}\right) \\
(\mathrm{dppb})(2)\end{array}$ & KOAc & DMA & 100 & 75 & \\
\hline $2^{[\mathrm{b}]}$ & $\begin{array}{c}\mathrm{PdCl}\left(\mathrm{C}_{3} \mathrm{H}_{5}\right) \\
(\mathrm{dppb})(2)\end{array}$ & KOAc & DMA & 100 & 74 & \\
\hline 3 & $\underset{(2)}{\mathrm{Pd}(\mathrm{OAc})_{2}}$ & KOAc & DMA & 100 & 82 & \\
\hline 4 & $\begin{array}{c}\mathrm{Pd}(\mathrm{OAc})_{2} \\
(1)\end{array}$ & KOAc & DMA & 100 & 85 & \\
\hline 5 & $\begin{array}{l}\mathrm{Pd}(\mathrm{OAc})_{2} \\
(0.5)\end{array}$ & KOAc & DMA & 83 & 79 & \\
\hline 6 & $\begin{array}{c}\mathrm{Pd}(\mathrm{OAc})_{2} \\
\text { (1) }\end{array}$ & PivOK & DMA & 80 & 53 & \\
\hline 7 & $\begin{array}{c}\mathrm{Pd}(\mathrm{OAc})_{2} \\
\text { (1) }\end{array}$ & $\mathrm{K}_{2} \mathrm{CO}_{3}$ & DMA & 95 & 12 & \\
\hline 8 & $\begin{array}{l}\mathrm{Pd}(\mathrm{OAc})_{2} \\
\text { (1) }\end{array}$ & $\mathrm{Cs}_{2} \mathrm{CO}_{3}$ & DMA & 67 & 0 & \\
\hline $9^{[b]}$ & $\begin{array}{c}\mathrm{Pd}(\mathrm{OAc})_{2} \\
(1)\end{array}$ & KOAc & DMA & 100 & 87 & \\
\hline $10^{[\mathrm{b}, \mathrm{c}]}$ & $\begin{array}{c}\mathrm{Pd}(\mathrm{OAc})_{2} \\
\text { (1) }\end{array}$ & KOAc & DMA & 100 & 89 & \\
\hline $11^{[\mathrm{b}]}$ & $\begin{array}{l}\mathrm{Pd}(\mathrm{OAc})_{2} \\
(1)\end{array}$ & KOAc & $\begin{array}{c}\text { CPM } \\
\text { E }\end{array}$ & 0 & 0 & \\
\hline $12^{[\mathrm{b}]}$ & $\begin{array}{l}\mathrm{Pd}(\mathrm{OAc})_{2} \\
(1)\end{array}$ & KOAc & $\begin{array}{c}\text { pentan- } \\
1-\mathrm{ol}\end{array}$ & 0 & 0 & \\
\hline $13^{[\mathrm{b}]}$ & $\begin{array}{l}\mathrm{Pd}(\mathrm{OAc})_{2} \\
(1)\end{array}$ & KOAc & $\mathrm{DEC}$ & 0 & 0 & \\
\hline $14^{[\mathrm{d}]}$ & $\mathrm{Pd}(\mathrm{OAc})_{2}$ & KOAc & DMA & 0 & 0 & \\
\hline
\end{tabular}

[a] Determined by GC-MS analysis using dodecane as internal standard. [b] Reaction performed at $120^{\circ} \mathrm{C}$. [c] Reaction performed using 1.15 equiv. of benzofurazan. [d] 4-Chlorobenzonitrile was used instead of 4-

bromobenzonitrile. DMA = Dimethylacetamide; $\mathrm{CPME}=$ Cyclopentylmethyl ether; DEC $=$ Diethylcarbonate

With the best conditions in hands, namely $1 \mathrm{~mol} \%$ phosphine-free $\mathrm{Pd}(\mathrm{OAc})_{2}$ associated to KOAc as base in DMA at $120^{\circ} \mathrm{C}$, we turned our attention to the scope of the reaction (Scheme 2). First, we examined the reactivity of para-substituted aryl bromides. The coupling of benzofurazan with 4-bromonitrobenzene, 4-bromobenzaldehyde, 4-bromopropiophenone, and ethyl 4-bromobenzoate proceeded nicely to afford $\mathbf{2}$ 5 in $69-83 \%$ yields. A moderate yield in the desired 4-aryl benzofurazan $\mathbf{6}$ was obtained from the less electron-deficient 4-bromochlorobenzene owing a partial conversion of this aryl bromide. For bromobenzene and electron-rich 4-bromoanisole, as their oxidative addition to palladium is more challenging, ${ }^{[16]}$ the reactions were conducted at a more elevated temperature of $150{ }^{\circ} \mathrm{C}$ to afford the 
desired products 7 and $\mathbf{8}$ in $65 \%$ and $62 \%$ yields, respectively. It should be noted that no additional phosphine ligand is required to perform such couplings. The electron-deficient meta-substituted aryl bromides 3-bromobenzonitrile, 3(trifluoromethyl)bromobenzene and 3bromonitrobenzene were also very reactive using 1 $\mathrm{mol} \% \mathrm{Pd}(\mathrm{OAc})_{2}$ as the catalyst at $120{ }^{\circ} \mathrm{C}$ and afforded 9-11 in yields of $71-79 \%$. Then, we investigated the effect of an ortho substituent on the aryl bromide. 2-Nitrile-, 2-formyl-, and 2-fluorobromobenzenes allowed the formation of the desired 4-arylated benzofurazans 12-14 in good yields. Under the standard reaction conditions, more sterically demanding 1-bromonaphthalene was also efficiently coupled with benzofurazan to deliver $\mathbf{1 5}$ in $57 \%$ yield. $N$-containing heteroaryl bromides (e.g., 2-bromo-6-(trifluoromethyl)pyridine, bromoquinoline, or 2-bromo-6-methoxypyridine) nicely reacted in the presence of $1 \mathrm{~mol} \% \mathrm{Pd}(\mathrm{OAc})_{2}$ at $150{ }^{\circ} \mathrm{C}$ to afford the desired products $\mathbf{1 6}-\mathbf{1 8}$ in good yields. In addition, this methodology allowed the synthesis 4-thiophen-2-ylfurazan $\mathbf{1 9}$, which is a useful intermediate in the design of organic materials, in $63 \%$ yield.

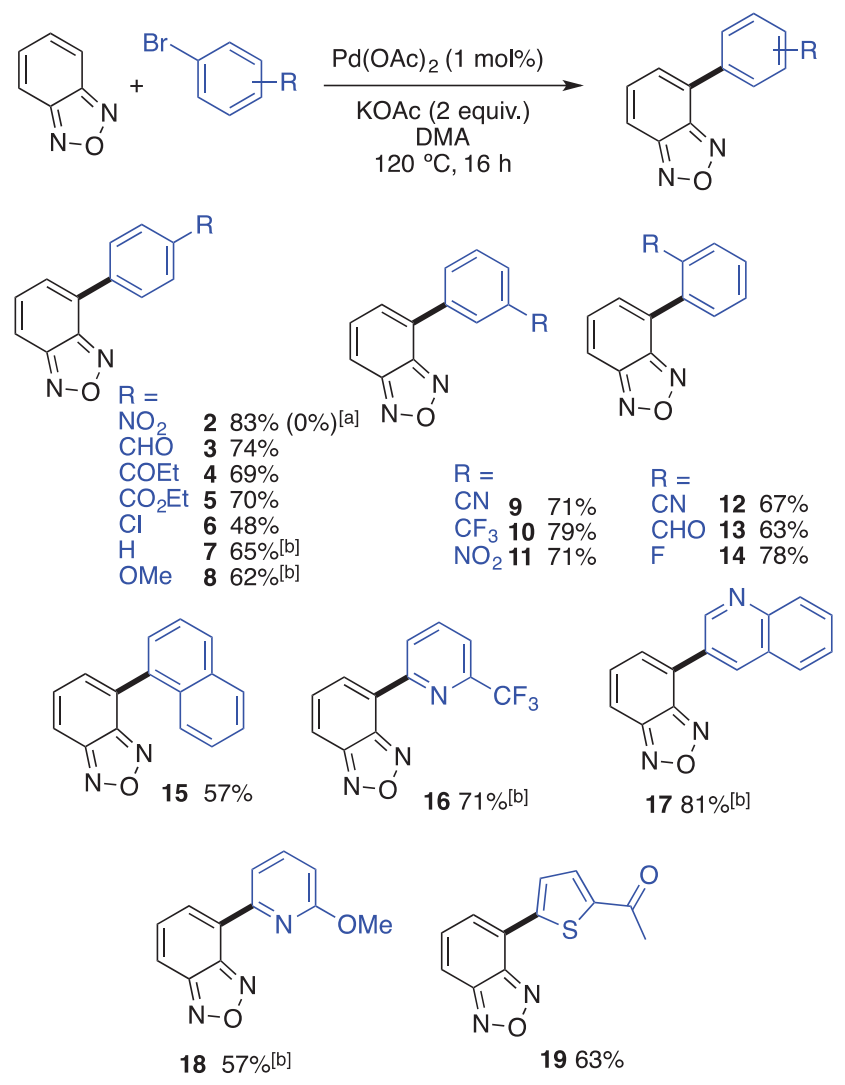

Scheme 2. Scope of Aryl Bromides in PalladiumCatalyzed Direct C4 Arylation of Benzofurazan. [a] 4Chloronitrobenzene was used instead of 4bromonitrobenzene. [b] Reaction performed at $150{ }^{\circ} \mathrm{C}$.

Next, we investigated the reactivity of 4- and 5substituted benzofurazans (Scheme 3). Benzofurazan substituted by an electron-donating group such as a methoxy at $\mathrm{C} 5$ position underwent $\mathrm{Pd}(\mathrm{OAc})_{2}$ catalyzed direct arylation. The reaction was completely regioselective with 4-bromobenzonitrile or 3-chlorobromobenzene and the arylation took place exclusively at the $\mathrm{C} 4$ position to afford products 20 and 21 in $68 \%$ and $56 \%$ yields, respectively (Scheme 3a). Conversely, the reaction was not regioselective using 2-bromonitrobenzene, probably because of its greater steric hindrance. The major regioisomer 22a was isolated in 38\% yield and resulted from the $\mathrm{C}-\mathrm{H}$ bond activation at the less sterically hindered $\mathrm{C} 7$ position of the benzofurazan (Scheme 1b); whereas, the $\mathrm{C} 4$-arylation regioisomer 22b was formed in less than $11 \%$ yield, making its isolation difficult. In contrast, benzofurazans substituted by an electron-withdrawing group such as 5-bromobenzofurazan or 4-nitrobenzofurazan were not reactive under our reaction conditions, even at $150{ }^{\circ} \mathrm{C}$ or in the presence of $\mathrm{PdCl}\left(\mathrm{C}_{3} \mathrm{H}_{5}\right)(\mathrm{dppb})$ catalyst (Scheme 3c and 3d).

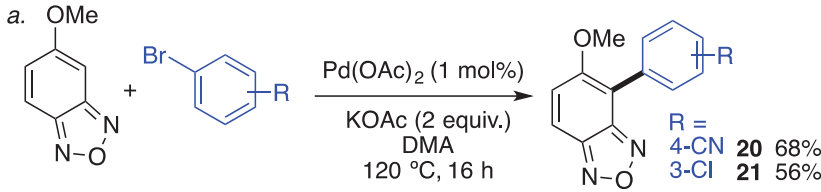

b.
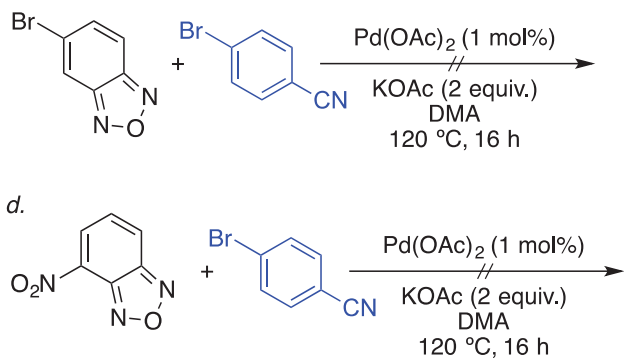

Scheme 3. Reactivities of Substituted Benzofurazans in Palladium-Catalyzed Direct Arylation.

Using the same reaction conditions, but in the presence of an excess amount of aryl bromides and base (3 equivalents), benzofurazan has been diarylated at both $\mathrm{C} 4$ and $\mathrm{C} 7$ carbons in one pot (Scheme 4). From bromobenzene, 4chlorobromobenzene and 3,5bis(trifluoromethyl)bromobenzene the diarylated products 23-26 were isolated in a range of yield of $57-72 \%$. Again, it is important to note that this procedure did not required the use of a phosphine ligand. 


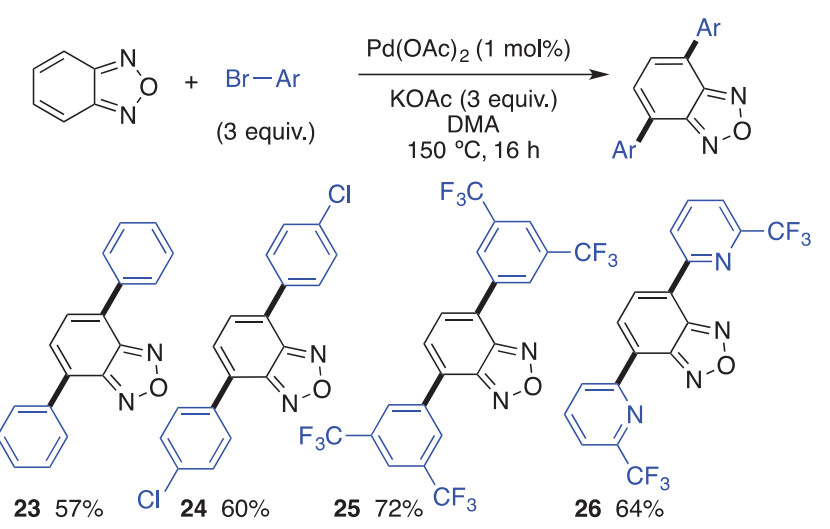

Scheme 4. Scope of Palladium-Catalyzed C4,C7 Diarylation of Benzofurazan

It is known that furazan unit of benzofurazans can easily undergo a ring opening in the presence of ethanolamines under acidic conditions to yield quinoxaline derivatives. ${ }^{[17]}$ Quinoxaline is an important motif, which is present in many pharmaceuticals. ${ }^{[18]}$ Therefore, we investigated the synthesis of some quinoxalines from arylated benzofurazans. Using the Samsonov conditions, ${ }^{[17]}$ namely, $10 \mathrm{~mol} \%$ para-toluenesulfonic acid in the presence of 1-aminoethanol, the arylated benzofurazans 6, 15 and 25 were converted into 5aryl quinoxalines $\mathbf{2 7 - 2 9}$ in $82-87 \%$ yields.

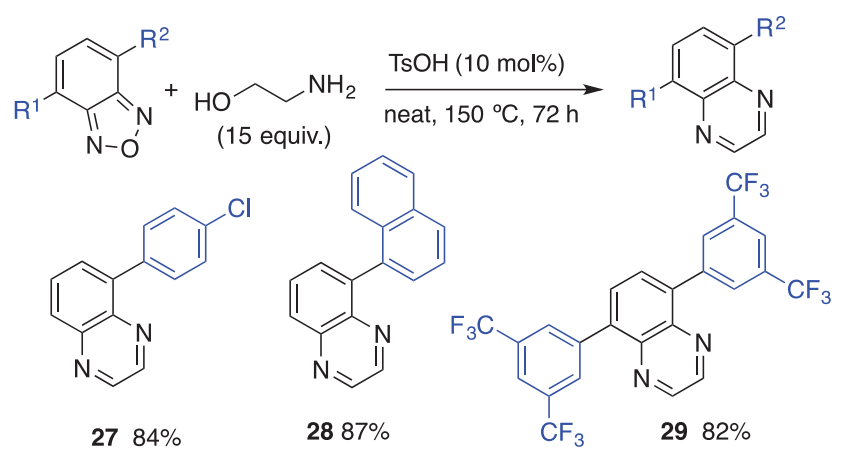

Scheme 5. Synthesis of 5-Arylated cc from Arylated Benzofurazans

\section{Conclusion}

In summary, we disclose here an elegant route to access 4-arylated benzofurazans from commercially available benzofurazan through palladium-catalyzed regioselective $\mathrm{C}-\mathrm{H}$ bond arylation. Our phosphinefree procedure using $\mathrm{Pd}(\mathrm{OAc})_{2}$ catalyst, $\mathrm{KOAc}$ as inexpensive base in the presence of aryl bromides as coupling partners promotes the $\mathrm{C} 4$ arylation or the $\mathrm{C} 4, \mathrm{C} 7$-diarylation of benzofurazan depending of the aryl bromide stoichiometry. A wide range of functions such as methoxy, fluoro, formyl, propionyl, carboxylate, nitrile or nitro on the aryl bromide is tolerated. Some sterically hindered aryl bromides, and sulfur or nitrogen containing heteroaromatic substrates have also been employed successfully. In addition, some of these 4-arylated benzofurazans were successfully transformed into quinoxaline derivatives.

\section{Experimental Section}

General Methods: All reactions were carried out under argon atmosphere with standard Schlenk techniques. DMA was purchased from Acros Organics and were not purified before use. Benzofurazans were purchased from Sigma-Aldrich. ${ }^{1} \mathrm{H}$ NMR spectra were recorded on Bruker GPX (400 MHz) spectrometer. Chemical shifts $(\delta)$ were reported in parts per million relative to residual chloroform (7.28 ppm for ${ }^{1} \mathrm{H} ; 77.23 \mathrm{ppm}$ for ${ }^{13} \mathrm{C}$ ), constants were reported in Hertz. ${ }^{1} \mathrm{H}$ NMR assignment abbreviations were the following: singlet $(\mathrm{s})$, doublet $(\mathrm{d})$, triplet $(\mathrm{t})$, quartet $(\mathrm{q})$, doublet of doublets (dd), doublet of triplets (dt), and multiplet (m). ${ }^{13} \mathrm{C}$ NMR spectra were recorded at 100 $\mathrm{MHz}$ on the same spectrometer and reported in ppm. All reagents were weighed and handled in air.

Procedure A (Palladium-catalyzed direct $\mathbf{C} 4$ arylation): To a $5 \mathrm{~mL}$ oven dried Schlenk tube, benzofurazan derivative $(1.15 \mathrm{mmol})$, aryl bromide $(1 \mathrm{mmol})$, AcOK (196 mg, $2 \mathrm{mmol})$, DMA (4 mL) and $\mathrm{Pd}(\mathrm{OAc})_{2}(2.2 \mathrm{mg}$, $0.01 \mathrm{mmol}, 2 \mathrm{~mol} \%$ ) were successively added. The reaction mixture was evacuated by vacuum-argon cycles ( 5 times) and stirred at $120-150{ }^{\circ} \mathrm{C}$ (oil bath temperature) for 16 hours (see tables and schemes). After cooling the reaction at room temperature and concentration, the crude mixture was purified by silica column chromatography to afford the desired arylated products.

4-(Benzo[c] $][1,2,5]$ oxadiazol-4-yl)benzonitrile (1): Following the general procedure A using 4bromobenzonitrile (182 $\mathrm{mg}, 1 \mathrm{mmol})$ and benzofurazan (138 $\mathrm{mg}, 1.15 \mathrm{mmol}$ ), the residue was purified by flash chromatography on silica gel (pentane- $\mathrm{CH}_{2} \mathrm{Cl}_{2}, 60-40$ ) to afford the desired compound $\mathbf{1}(197 \mathrm{mg}, 89 \%)$ as a white solid $\left(\mathrm{Mp}=180-184{ }^{\circ} \mathrm{C}\right) .{ }^{1} \mathrm{H}$ NMR $\left(400 \mathrm{MHz}, \mathrm{CDCl}_{3}\right) \delta$ (ppm) 8.13 (d, $J=8.5 \mathrm{~Hz}, 2 \mathrm{H}), 7.91(\mathrm{~d}, J=8.9 \mathrm{~Hz}, 1 \mathrm{H})$, $7.82(\mathrm{~d}, J=8.5 \mathrm{~Hz}, 2 \mathrm{H}), 7.66(\mathrm{~d}, J=7.0 \mathrm{~Hz}, 1 \mathrm{H}), 7.56(\mathrm{dd}$, $J=7.0,9.0 \mathrm{~Hz}, 1 \mathrm{H}) .{ }^{13} \mathrm{C} \mathrm{NMR}\left(100 \mathrm{MHz}, \mathrm{CDCl}_{3}\right) \delta(\mathrm{ppm})$ $149.7,148.1,139.4,132.7,131.6,129.3,128.9,128.4$, $118.5,116.8,112.8$.Elemental analysis: calcd (\%) for $\mathrm{C}_{13} \mathrm{H}_{7} \mathrm{~N}_{3} \mathrm{O}$ (221.22): C 70.58, H 3.19; found: C 70.85, H 3.46 .

4-(4-Nitrophenyl)benzo[c][1,2,5]oxadiazole (2): Following the general procedure A using 4bromonitrobenzene $(202 \mathrm{mg}, 1 \mathrm{mmol})$ and benzofurazan (138 $\mathrm{mg}, 1.15 \mathrm{mmol}$ ), the residue was purified by flash chromatography on silica gel (pentane-EtOAc, 90-10) to afford the desired compound $2(200 \mathrm{mg}, 83 \%)$ as a yellow solid $\left(\mathrm{MP}=198-202{ }^{\circ} \mathrm{C}\right) .{ }^{1} \mathrm{H}$ NMR $\left(400 \mathrm{MHz}, \mathrm{CDCl}_{3}\right) \delta$ (ppm) 8.42 (d, $J=8.9 \mathrm{~Hz}, 2 \mathrm{H}), 8.23$ (d, $J=8.9 \mathrm{~Hz}, 2 \mathrm{H})$, $7.96(\mathrm{~d}, J=9.0 \mathrm{~Hz}, 1 \mathrm{H}), 7.73(\mathrm{~d}, J=6.8 \mathrm{~Hz}, 1 \mathrm{H}), 7.60$ (dd, $J=6.8,9.0 \mathrm{~Hz}, 1 \mathrm{H})$.

${ }^{13} \mathrm{C}$ NMR $\left(100 \mathrm{MHz}, \mathrm{CDCl}_{3}\right) \delta$ (ppm) 149.7, 148.2, 148.1, 141.2, 131.5, 129.7, 129.2, 128.1, 124.2, 117.1.Elemental analysis: calcd (\%) for $\mathrm{C}_{12} \mathrm{H}_{7} \mathrm{~N}_{3} \mathrm{O}_{3}$ (241.21): C 59.75, $\mathrm{H}$ 2.93; found: C 59.81, H 3.19. 
4-(Benzo[c] $[1,2,5]$ oxadiazol-4-yl)benzaldehyde (3): Following the general procedure A using 4bromobenzaldehyde (185 $\mathrm{mg}, 1 \mathrm{mmol})$ and benzofurazan (138 $\mathrm{mg}, 1.15 \mathrm{mmol}$ ), the residue was purified by flash chromatography on silica gel (pentane- $\mathrm{Et}_{2} \mathrm{O}, 70-30$ ) to afford the desired compound 3 (166 $\mathrm{mg}, 74 \%$ ) as a pale yellow solid $\left(\mathrm{MP}=128-130{ }^{\circ} \mathrm{C}\right) .{ }^{1} \mathrm{H}$ NMR $(400 \mathrm{MHz}$, $\left.\mathrm{CDCl}_{3}\right) \delta(\mathrm{ppm}) 10.14(\mathrm{~s}, 1 \mathrm{H}), 8.22(\mathrm{~d}, J=8.4 \mathrm{~Hz}, 2 \mathrm{H})$, 8.07 (d, $J=8.4 \mathrm{~Hz}, 2 \mathrm{H}), 7.93(\mathrm{~d}, J=9.0 \mathrm{~Hz}, 1 \mathrm{H}), 7.72(\mathrm{~d}$, $J=6.8 \mathrm{~Hz}, 1 \mathrm{H}), 7.58(\mathrm{dd}, J=6.8,9.0 \mathrm{~Hz}, 1 \mathrm{H}) .{ }^{13} \mathrm{C} \mathrm{NMR}$ $\left(100 \mathrm{MHz}_{\mathrm{CDCl}}\right) \delta(\mathrm{ppm}) 191.6,149.8,148.3,140.9$, $136.5,131.6,130.2,129.3,129.1,129.0,116.5$.Elemental analysis: calcd (\%) for $\mathrm{C}_{13} \mathrm{H}_{8} \mathrm{~N}_{2} \mathrm{O}_{2}$ (224.21): $\mathrm{C}$ 69.64, $\mathrm{H}$ 3.60; found: C 69.52, H 3.75.

\section{1-(4-(Benzo[c] $[1,2,5]$ oxadiazol-4-}

yl)phenyl)propan-1-one (4): Following the general procedure A using 4-bromopropiophenone (213 mg, 1 $\mathrm{mmol})$ and benzofurazan $(138 \mathrm{mg}, 1.15 \mathrm{mmol})$, the residue was purified by flash chromatography on silica gel (pentane- $\mathrm{CH}_{2} \mathrm{Cl}_{2}, 50-50$ ) to afford the desired compound 4 $(174 \mathrm{mg}, 69 \%)$ as a white solid $\left(\mathrm{MP}=161-165{ }^{\circ} \mathrm{C}\right) .{ }^{1} \mathrm{H}$ NMR (400 MHz, CDCl 3 ) $\delta(\mathrm{ppm}) 8.14(\mathrm{~s}, 4 \mathrm{H}), 7.90(\mathrm{~d}, J=$ $9.0 \mathrm{~Hz}, 1 \mathrm{H}), 7.69$ (d, $J=6.8 \mathrm{~Hz}, 1 \mathrm{H}), 7.57$ (dd, $J=6.8,9.0$ $\mathrm{Hz}, 1 \mathrm{H}), 3.09$ (q, $J=7.3 \mathrm{~Hz}, 2 \mathrm{H}), 1.29$ (t, $J=7.2 \mathrm{~Hz}$, $3 \mathrm{H}) .{ }^{13} \mathrm{C}$ NMR $\left(100 \mathrm{MHz}, \mathrm{CDCl}_{3}\right) \delta$ (ppm) 200.2, 149.8, $148.4,139.3,137.1,131.6,129.3,128.9,128.6,128.5$, 116.1, 32.0, 8.2.Elemental analysis: calcd (\%) for $\mathrm{C}_{15} \mathrm{H}_{12} \mathrm{~N}_{2} \mathrm{O}_{2}$ (252.27): C 71.42, $\mathrm{H} 4.79$; found: $\mathrm{C} 71.56, \mathrm{H}$ 3.81 .

Ethyl 4-(benzo $[c][1,2,5]$ oxadiazol-4-yl)benzoate (5): Following the general procedure A using ethyl 4bromobenzoate (229 $\mathrm{mg}, 1 \mathrm{mmol})$ and benzofurazan (138 $\mathrm{mg}, 1.15 \mathrm{mmol})$, the residue was purified by flash chromatography on silica gel (pentane- $\mathrm{CH}_{2} \mathrm{Cl}_{2}, 40-60$ ) to afford the desired compound $\mathbf{5}(188 \mathrm{mg}, 70 \%)$ as an orange solid $\left(\mathrm{MP}=105-108{ }^{\circ} \mathrm{C}\right) .{ }^{1} \mathrm{H}$ NMR $\left(400 \mathrm{MHz}, \mathrm{CDCl}_{3}\right) \delta$ (ppm) 8.22 (d, $J=8.2 \mathrm{~Hz}, 2 \mathrm{H}), 8.11$ (d, $J=8.2 \mathrm{~Hz}, 2 \mathrm{H})$, $7.89(\mathrm{~d}, J=9.0 \mathrm{~Hz}, 1 \mathrm{H}), 7.68(\mathrm{~d}, J=6.8 \mathrm{~Hz}, 1 \mathrm{H}), 7.56(\mathrm{dd}$, $J=6.8,9.0 \mathrm{~Hz}, 1 \mathrm{H}), 4.45(\mathrm{q}, J=7.1 \mathrm{~Hz}, 2 \mathrm{H}), 1.46(\mathrm{t}, J=$ $7.1 \mathrm{~Hz}, 3 \mathrm{H}) .{ }^{13} \mathrm{C}$ NMR $\left(100 \mathrm{MHz}, \mathrm{CDCl}_{3}\right) \delta$ (ppm) 166.1, $149.8,148.4,139.3,131.6,131.0,130.1,129.4,128.9$, $128.3,116.0,61.2,14.4$.

Elemental analysis: calcd (\%) for $\mathrm{C}_{15} \mathrm{H}_{12} \mathrm{~N}_{2} \mathrm{O}_{3}$ (268.27): C 67.16, H 4.51; found: C 66.98, H 4.79.

4-(4-Chlorophenyl)benzo[c] $[1,2,5]$ oxadiazole (6): Following the general procedure A using 1-bromo-4chlorobenzene (191 mg, $1 \mathrm{mmol})$ and benzofurazan (138 $\mathrm{mg}, 1.15 \mathrm{mmol})$, the residue was purified by flash chromatography on silica gel (pentane- $\mathrm{CH}_{2} \mathrm{Cl}_{2}, 80-20$ ) to afford the desired compound $6(111 \mathrm{mg}, 48 \%)$ as a yellow solid $\left(\mathrm{MP}=95-97{ }^{\circ} \mathrm{C}\right) . \quad{ }^{1} \mathrm{H}$ NMR $\left(400 \mathrm{MHz}, \mathrm{CDCl}_{3}\right) \delta$ (ppm) $7.98(\mathrm{~d}, J=8.5 \mathrm{~Hz}, 2 \mathrm{H}), 7.85$ (d, $J=8.9 \mathrm{~Hz}, 1 \mathrm{H})$, $7.59(\mathrm{~d}, J=6.8 \mathrm{~Hz}, 1 \mathrm{H}), 7.57-7.44(\mathrm{~m}, 3 \mathrm{H}) .{ }^{13} \mathrm{C} \mathrm{NMR}$ $\left(100 \mathrm{MHz}, \mathrm{CDCl}_{3}\right) \delta(\mathrm{ppm}) 149.8,148.4,135.4,133.6$, 131.7, 129.6, 129.2, 129.2, 128.0, 115.5. Elemental analysis: calcd (\%) for $\mathrm{C}_{12} \mathrm{H}_{7} \mathrm{ClN}_{2} \mathrm{O}$ (230.65): $\mathrm{C} 62.49, \mathrm{H}$ 3.06; found: C $62.53, \mathrm{H} 3.00$.

4-(Phenyl)benzo[c][1,2,5]oxadiazole (7): Following the general procedure A using bromobenzene (157 mg, 1 $\mathrm{mmol}$ ) and benzofurazan $(138 \mathrm{mg}, 1.15 \mathrm{mmol})$, the residue was purified by flash chromatography on silica gel (pentane-toluene, 80-20) to afford the desired compound 7 $(127 \mathrm{mg}, 65 \%)$ as a yellow solid $\left(\mathrm{MP}=58-60{ }^{\circ} \mathrm{C}\right) .{ }^{1} \mathrm{H}$ NMR (400 MHz, $\left.\mathrm{CDCl}_{3}\right) \delta(\mathrm{ppm}) 8.04-7.98(\mathrm{~m}, 2 \mathrm{H})$, $7.87-7.80(\mathrm{~m}, 1 \mathrm{H}), 7.60(\mathrm{td}, J=1.0,6.8 \mathrm{~Hz}, 1 \mathrm{H}), 7.57-$ $7.46(\mathrm{~m}, 4 \mathrm{H}) .{ }^{13} \mathrm{C}$ NMR $\left(100 \mathrm{MHz}, \mathrm{CDCl}_{3}\right) \delta(\mathrm{ppm}) 149.8$, $148.6,135.3,131.8,130.5,129.3,129.0,128.4,128.0$, 115.0. Elemental analysis: calcd (\%) for $\mathrm{C}_{12} \mathrm{H}_{8} \mathrm{~N}_{2} \mathrm{O}$ (196.21): C 73.46, H 4.11; found: C 73.69, H 3.90.

4-(4-methoxyphenyl)benzo $[c][1,2,5]$ oxadiazole $(8)$ : Following the general procedure A using 4-bromoanisole (187 $\mathrm{mg}, 1 \mathrm{mmol})$ and benzofurazan $(138 \mathrm{mg}, 1.15 \mathrm{mmol})$, the residue was purified by flash chromatography on silica gel (pentane-toluene, 80-20) to afford the desired compound $8(140 \mathrm{mg}, 62 \%)$ as an orange solid (MP $=118$ $\left.121{ }^{\circ} \mathrm{C}\right) .{ }^{1} \mathrm{H}$ NMR $\left(400 \mathrm{MHz}, \mathrm{CDCl}_{3}\right) \delta(\mathrm{ppm}) 8.00(\mathrm{~d}, J=$ $8.8 \mathrm{~Hz}, 2 \mathrm{H}), 7.77$ (dd, $J=1.3,8.5 \mathrm{~Hz}, 1 \mathrm{H}), 7.58-7.47$ (m, 2H), $7.08(\mathrm{~d}, J=8.8 \mathrm{~Hz}, 2 \mathrm{H}), 3.92(\mathrm{~s}, 3 \mathrm{H}) .{ }^{13} \mathrm{C}$ NMR $(100$ $\left.\mathrm{MHz}, \mathrm{CDCl}_{3}\right) \delta(\mathrm{ppm}) 160.5,149.9,148.7,131.9,130.1$, $129.7,127.7,126.7,114.4,114.1,55.4$. This is a known compound and the spectral data are identical to those reported in literature. ${ }^{[5 a]}$

3-(Benzo[c] $[1,2,5]$ oxadiazol-4-yl)benzonitrile (9): Following the general procedure A using 3bromobenzonitrile $(182 \mathrm{mg}, 1 \mathrm{mmol})$ and benzofurazan (138 $\mathrm{mg}, 1.15 \mathrm{mmol}$ ), the residue was purified by flash chromatography on silica gel (pentane-toluene, 80-20) to afford the desired compound $9(157 \mathrm{mg}, 71 \%)$ as a yellow solid $\left(\mathrm{MP}=156-159{ }^{\circ} \mathrm{C}\right) .{ }^{1} \mathrm{H}$ NMR $\left(400 \mathrm{MHz}, \mathrm{CDCl}_{3}\right) \delta$ (ppm) $\delta 8.34-8.24(\mathrm{~m}, 2 \mathrm{H}), 7.92(\mathrm{~d}, J=8.9 \mathrm{~Hz}, 1 \mathrm{H}), 7.78$ $(\mathrm{dt}, J=1.4,7.7 \mathrm{~Hz}, 1 \mathrm{H}), 7.69$ (d, $J=7.8 \mathrm{~Hz}, 1 \mathrm{H}), 7.67-$ $7.63(\mathrm{~m}, 1 \mathrm{H}), 7.58(\mathrm{dd}, J=6.8,8.9 \mathrm{~Hz}, 1 \mathrm{H}) .{ }^{13} \mathrm{C} \mathrm{NMR}$ $\left(100 \mathrm{MHz}, \mathrm{CDCl}_{3}\right) \delta(\mathrm{ppm}) 149.7,148.2,136.4,132.6$, $132.5,131.8,131.6,129.9,128.9,128.1,118.4,116.5$, 113.4. Elemental analysis: calcd $(\%)$ for $\mathrm{C}_{13} \mathrm{H}_{7} \mathrm{~N}_{3} \mathrm{O}$ (221.22): C 70.58, H 3.19; found: C 70.41, H 3.29.

$$
\text { 4-(3- }
$$

\section{(Trifluoromethyl)phenyl)benzo[c][1,2,5]oxadiazole}

(10): Following the general procedure A using 1-bromo-3(trifluoromethyl)benzene $(225 \mathrm{mg}, 1 \mathrm{mmol})$ and benzofurazan (138 $\mathrm{mg}, 1.15 \mathrm{mmol})$, the residue was purified by flash chromatography on silica gel (pentanetoluene, 90-10) to afford the desired compound 10 (209 $\mathrm{mg}, 79 \%)$ as pale yellow oil. ${ }^{1} \mathrm{H}$ NMR (400 $\left.\mathrm{MHz}, \mathrm{CDCl}_{3}\right)$ $\delta(\mathrm{ppm}) 8.28-8.24(\mathrm{~m}, 2 \mathrm{H}), 7.90(\mathrm{~d}, J=8.9 \mathrm{~Hz}, 1 \mathrm{H}), 7.77$ $-7.73(\mathrm{~m}, 1 \mathrm{H}), 7.70(\mathrm{~d}, J=7.9 \mathrm{~Hz}, 1 \mathrm{H}), 7.67(\mathrm{~d}, J=6.8$ $\mathrm{Hz}, 1 \mathrm{H}), 7.57(\mathrm{dd}, J=6.8,9.0 \mathrm{~Hz}, 1 \mathrm{H}) .{ }^{13} \mathrm{C}$ NMR $(100$ $\left.\mathrm{MHz}, \mathrm{CDCl}_{3}\right) \delta(\mathrm{ppm}) 149.7,148.4,135.9,131.7,131.6$, $131.5(\mathrm{q}, J=32.8 \mathrm{~Hz}), 129.5,129.0,128.7,125.9$ (q, $J=$ $4.1 \mathrm{~Hz}), 125.0(\mathrm{q}, J=4.1 \mathrm{~Hz}), 123.9(\mathrm{q}, J=272.4), 116.1$. Elemental analysis: calcd (\%) for $\mathrm{C}_{13} \mathrm{H}_{7} \mathrm{~F}_{3} \mathrm{~N}_{2} \mathrm{O}$ (264.21): C 59.10, H 2.67; found: C 59.33, H 2.51.

4-(3-Nitrophenyl)benzo[c] $[1,2,5]$ oxadiazole (11): Following the general procedure A using 3bromonitrobenzene (202 $\mathrm{mg}, 1 \mathrm{mmol})$ and benzofurazan (138 $\mathrm{mg}, 1.15 \mathrm{mmol}$ ), the residue was purified by flash chromatography on silica gel (pentane-EtOAc, 90-1510 to afford the desired compound $\mathbf{1 1}(171 \mathrm{mg}, 71 \%)$ as a brown solid $\left(\mathrm{MP}=150-153{ }^{\circ} \mathrm{C}\right) .{ }^{1} \mathrm{H}$ NMR $\left(400 \mathrm{MHz}, \mathrm{CDCl}_{3}\right) \delta$ (ppm) $8.86(\mathrm{~s}, 1 \mathrm{H}), 8.45(\mathrm{~d}, J=7.5 \mathrm{~Hz}, 1 \mathrm{H}), 8.35(\mathrm{~d}, J=$ $7.9 \mathrm{~Hz}, 1 \mathrm{H}), 7.94(\mathrm{~d}, J=9.1 \mathrm{~Hz}, 1 \mathrm{H}), 7.81-7.68(\mathrm{~m}, 2 \mathrm{H})$, $7.64-7.53(\mathrm{~m}, 1 \mathrm{H}) .{ }^{13} \mathrm{C}$ NMR $\left(100 \mathrm{MHz}, \mathrm{CDCl}_{3}\right) \delta(\mathrm{ppm})$ $149.7,148.8,148.2,136.7,134.4,131.6,130.1,129.1$, $127.9,123.8,123.0,116.7$. Elemental analysis: calcd (\%) for $\mathrm{C}_{12} \mathrm{H}_{7} \mathrm{~N}_{3} \mathrm{O}_{3}$ (241.21): C 59.75, H 2.93; found: C 59.98, H 3.07.

2-(Benzo[c][1,2,5]oxadiazol-4-yl)benzonitrile (12): Following the general procedure A using 2bromobenzonitrile $(182 \mathrm{mg}, 1 \mathrm{mmol})$ and benzofurazan (138 $\mathrm{mg}, 1.15 \mathrm{mmol}$ ), the residue was purified by flash 
chromatography on silica gel (pentane- $\mathrm{CH}_{2} \mathrm{Cl}_{2}, 60-40$ ) to afford the desired compound $\mathbf{1 2}(148 \mathrm{mg}, 67 \%)$ as a white solid $\left(\mathrm{MP}=164-166{ }^{\circ} \mathrm{C}\right) .{ }^{1} \mathrm{H}$ NMR $\left(400 \mathrm{MHz}, \mathrm{CDCl}_{3}\right) \delta$ (ppm) 7.97 (d, $J=9.1 \mathrm{~Hz}, 1 \mathrm{H}), 7.93(\mathrm{~d}, J=7.8 \mathrm{~Hz}, 1 \mathrm{H})$, $7.90(\mathrm{~d}, J=7.2 \mathrm{~Hz}, 1 \mathrm{H}), 7.79(\mathrm{dt}, J=1.4,7.7 \mathrm{~Hz}, 1 \mathrm{H})$, $7.72(\mathrm{~d}, J=6.7 \mathrm{~Hz}, 1 \mathrm{H}), 7.65-7.57(\mathrm{~m}, 2 \mathrm{H}) .{ }^{13} \mathrm{C} \mathrm{NMR}$ $\left(100 \mathrm{MHz}, \mathrm{CDCl}_{3}\right) \delta(\mathrm{ppm}) 149.5,148.6,138.7,134.1$, $133.0,131.7,131.4,131.1,129.3,126.9,118.0,117.0$, 111.7. Elemental analysis: calcd $(\%)$ for $\mathrm{C}_{13} \mathrm{H}_{7} \mathrm{~N}_{3} \mathrm{O}$ (221.22): C 70.58, H 3.19; found: C 70.39, H 3.30.

\section{2-(Benzo[c][1,2,5]oxadiazol-4-yl)benzaldehyde}

(13): Following the general procedure A using 2bromobenzaldehyde (185 $\mathrm{mg}, 1 \mathrm{mmol})$ and benzofurazan (138 $\mathrm{mg}, 1.15 \mathrm{mmol}$ ), the residue was purified by flash chromatography on silica gel (pentane- $\mathrm{Et}_{2} \mathrm{O}, 70-30$ ) to afford the desired compound 13 (141 $\mathrm{mg}, 63 \%$ ) as an orange solid $\left(\mathrm{MP}=140-143{ }^{\circ} \mathrm{C}\right) .{ }^{1} \mathrm{H} \mathrm{NMR}(400 \mathrm{MHz}$, $\left.\mathrm{CDCl}_{3}\right) \delta(\mathrm{ppm}) 9.97(\mathrm{~s}, 1 \mathrm{H}), 8.12(\mathrm{~d}, J=7.9 \mathrm{~Hz}, 1 \mathrm{H}), 7.94$ $(\mathrm{d}, J=9.0 \mathrm{~Hz}, 1 \mathrm{H}), 7.77(\mathrm{dt}, J=1.5,7.5 \mathrm{~Hz}, 1 \mathrm{H}), 7.71-$ $7.63(\mathrm{~m}, 2 \mathrm{H}), 7.57(\mathrm{dd}, J=6.6,9.1 \mathrm{~Hz}, 1 \mathrm{H}), 7.36(\mathrm{~d}, J=$ $6.6 \mathrm{~Hz}, 1 \mathrm{H}) .{ }^{13} \mathrm{C} \mathrm{NMR}\left(100 \mathrm{MHz}, \mathrm{CDCl}_{3}\right) \delta(\mathrm{ppm}) 190.8$, $149.6,149.1,138.1,134.4,133.9,131.8,131.3,131.2$, $129.7,129.5,128.0,116.4$. Elemental analysis: calcd (\%) for $\mathrm{C}_{13} \mathrm{H}_{8} \mathrm{~N}_{2} \mathrm{O}_{2}$ (224.21): C 69.64, H 3.60; found: C 69.72, H 3.88 .

4-(2-Fluorophenyl)benzo[c][1,2,5]oxadiazole (14): Following the general procedure A using 1-bromo-2fluorobenzene $(175 \mathrm{mg}, 1 \mathrm{mmol})$ and benzofurazan (138 $\mathrm{mg}, 1.15 \mathrm{mmol})$, the residue was purified by flash chromatography on silica gel (pentane-toluene, 90-10) to afford the desired compound $14(167 \mathrm{mg}, 78 \%)$ as a yellow solid $\left(\mathrm{MP}=119-121{ }^{\circ} \mathrm{C}\right) .{ }^{1} \mathrm{H}$ NMR $\left(400 \mathrm{MHz}, \mathrm{CDCl}_{3}\right) \delta$ (ppm) $7.91-7.84(\mathrm{~m}, 2 \mathrm{H}), 7.62(\mathrm{~d}, J=6.9 \mathrm{~Hz}, 1 \mathrm{H}), 7.54$ $(\mathrm{dd}, J=6.8,9.0 \mathrm{~Hz}, 1 \mathrm{H}), 7.51-7.44(\mathrm{~m}, 1 \mathrm{H}), 7.34(\mathrm{td}, J=$ 1.2, 7.6 Hz, 1H), $7.30-7.24(\mathrm{~m}, 1 \mathrm{H}) .{ }^{13} \mathrm{C}$ NMR $(100$ $\left.\mathrm{MHz}, \mathrm{CDCl}_{3}\right) \delta(\mathrm{ppm}) 159.9(\mathrm{~d}, J=249.5 \mathrm{~Hz}), 149.5$, 148.7, 131.5, $131.2(\mathrm{~d}, J=5.4 \mathrm{~Hz}), 130.8(\mathrm{~d}, J=8.6 \mathrm{~Hz})$, $124.9,124.5,124.5,123.1$ (d, $J=12,8 \mathrm{~Hz}), 116.4$ (d, $J=$ $20.9 \mathrm{~Hz})$, 115.8. Elemental analysis: calcd (\%) for $\mathrm{C}_{12} \mathrm{H}_{7} \mathrm{FN}_{2} \mathrm{O}$ (214.20): C 67.29, H 3.29; found: C 67.18, H 3.45 .

4-(Naphthalen-1-yl)benzo $[c][1,2,5]$ oxadiazole (15): Following the general procedure A using 1bromonaphthalene $(207 \mathrm{mg}, 1 \mathrm{mmol})$ and benzofurazan (138 $\mathrm{mg}, 1.15 \mathrm{mmol}$ ), the residue was purified by flash chromatography on silica gel (pentane-toluene, 95-15) to afford the desired compound $\mathbf{1 5}(140 \mathrm{mg}, 57 \%)$ as a yellow solid $\left(\mathrm{MP}=68-71{ }^{\circ} \mathrm{C}\right) .{ }^{1} \mathrm{H}$ NMR $\left(400 \mathrm{MHz}, \mathrm{CDCl}_{3}\right) \delta$ (ppm) $8.03-7.96(\mathrm{~m}, 2 \mathrm{H}), 7.94(\mathrm{~d}, J=8.9 \mathrm{~Hz}, 1 \mathrm{H}), 7.74$ $(\mathrm{d}, J=8.9 \mathrm{~Hz}, 1 \mathrm{H}), 7.69-7.63(\mathrm{~m}, 1 \mathrm{H}), 7.63-7.56(\mathrm{~m}$, 2H), $7.55-7.50(\mathrm{~m}, 2 \mathrm{H}), 7.50-7.43(\mathrm{~m}, 1 \mathrm{H}) .{ }^{13} \mathrm{C} \mathrm{NMR}$ $\left(100 \mathrm{MHz}, \mathrm{CDCl}_{3}\right) \delta(\mathrm{ppm}) 149.9,149.5,133.9,133.5$, $131.6,131.6,131.3,130.1,129.5,128.6,127.9$, 126.6, $126.2,125.3,125.3,115.5$. Elemental analysis: calcd (\%) for $\mathrm{C}_{16} \mathrm{H}_{10} \mathrm{~N}_{2} \mathrm{O}$ (246.27): C 78.03, H 4.09; found: C 78.45, H 4.19 .

\section{4-(6-(Trifluoromethyl)pyridin-2-}

yl)benzo[c] $[1,2,5]$ oxadiazole (16): Following the general procedure A using 2-bromo-6-(trifluoromethyl)pyridine (225 mg, $1 \mathrm{mmol}$ ) and benzofurazan (138 mg, $1.15 \mathrm{mmol})$, the residue was purified by flash chromatography on silica gel (pentane-toluene, 80-20) to afford the desired compound 16 (188 mg, 71\%) as a yellow solid (MP $=148$ $\left.150{ }^{\circ} \mathrm{C}\right) .{ }^{1} \mathrm{H}$ NMR $\left(400 \mathrm{MHz}, \mathrm{CDCl}_{3}\right) \delta(\mathrm{ppm}) 8.89(\mathrm{~d}, J=$ $8.0 \mathrm{~Hz}, 1 \mathrm{H}), 8.64(\mathrm{~d}, J=6.9 \mathrm{~Hz}, 1 \mathrm{H}), 8.15-8.05(\mathrm{~m}, 1 \mathrm{H})$, $7.97(\mathrm{dd}, J=0.8,8.9 \mathrm{~Hz}, 1 \mathrm{H}), 7.74(\mathrm{dd}, J=0.9,7.8 \mathrm{~Hz}$, $1 \mathrm{H}), 7.64(\mathrm{dd}, J=6.9,9.0 \mathrm{~Hz}, 1 \mathrm{H})$.

${ }^{13} \mathrm{C}$ NMR $\left(100 \mathrm{MHz}, \mathrm{CDCl}_{3}\right) \delta(\mathrm{ppm}) 152.1,149.9,148.3$ $(\mathrm{q}, J=34.9 \mathrm{~Hz}), 147.7,138.8,131.9,131.2,126.8,126.5$, $121.4(\mathrm{q}, J=274.7 \mathrm{~Hz}), 119.9,117.7$. Elemental analysis: calcd (\%) for $\mathrm{C}_{12} \mathrm{H}_{6} \mathrm{~F}_{3} \mathrm{~N}_{3} \mathrm{O}$ (265.20): C 54.35, H 2.28; found: C 64.43, $\mathrm{H} 2.37$.

4-(Quinolin-3-yl)benzo[c] $[1,2,5]$ oxadiazole (17): Following the general procedure A using 3bromoquinoline (208 mg, $1 \mathrm{mmol}$ ) and benzofurazan (138 $\mathrm{mg}, 1.15 \mathrm{mmol})$, the residue was purified by flash chromatography on silica gel (pentane-EtOAc, 70-30) to afford the desired compound 17 (200 mg, 81\%) as an orange solid $\left(\mathrm{MP}=143-145{ }^{\circ} \mathrm{C}\right) .{ }^{1} \mathrm{H}$ NMR $(400 \mathrm{MHz}$, DMSO- $\left.d_{6}\right) \delta(\mathrm{ppm}) 9.53(\mathrm{~d}, J=2.4 \mathrm{~Hz}, 1 \mathrm{H}), 9.06(\mathrm{~d}, J=$ $2.0 \mathrm{~Hz}, 1 \mathrm{H}), 8.18-8.10(\mathrm{~m}, 4 \mathrm{H}), 7.87(\mathrm{ddd}, J=1.4,6.9$, $8.4 \mathrm{~Hz}, 1 \mathrm{H}), 7.81(\mathrm{dd}, J=6.8,9.0 \mathrm{~Hz}, 1 \mathrm{H}), 7.72(\mathrm{ddd}, J=$ $1.2,6.9,8.1 \mathrm{~Hz}, 1 \mathrm{H}) .{ }^{13} \mathrm{C}$ NMR $\left(100 \mathrm{MHz}, \mathrm{DMSO}-d_{6}\right) \delta$ (ppm) 150.1, 150.0, 148.7, 147.8, 135.9, 133.6, 131.0, $130.9,129.3,129.2,128.3,127.9,127.7,126.3,116.3$. Elemental analysis: calcd (\%) for $\mathrm{C}_{15} \mathrm{H}_{9} \mathrm{~N}_{3} \mathrm{O}$ (247.26): C 72.87, H 3.67; found: C 72.98, H 3.54.

\section{4-(6-Methoxypyridin-2-}

yl)benzo[c] $[1,2,5]$ oxadiazole (18): Following the general procedure A using 2-bromo-6-methoxypyridine (188 mg, 1 $\mathrm{mmol})$ and benzofurazan $(138 \mathrm{mg}, 1.15 \mathrm{mmol})$, the residue was purified by flash chromatography on silica gel (pentane-toluene, 90-10) to afford the desired compound $18(130 \mathrm{mg}, 57 \%)$ as a yellow solid $\left(\mathrm{MP}=140-143{ }^{\circ} \mathrm{C}\right) .{ }^{1} \mathrm{H}$ NMR $\left(400 \mathrm{MHz}, \mathrm{CDCl}_{3}\right) \delta(\mathrm{ppm}) 8.53(\mathrm{~d}, J=6.9 \mathrm{~Hz}, 1 \mathrm{H})$, $8.32(\mathrm{~d}, J=7.5 \mathrm{~Hz}, 1 \mathrm{H}), 7.89$ (d, $J=8.9 \mathrm{~Hz}, 1 \mathrm{H}), 7.79$ (dd, $J=7.4,8.3 \mathrm{~Hz}, 1 \mathrm{H}), 7.60(\mathrm{dd}, J=6.9,9.0 \mathrm{~Hz}, 1 \mathrm{H}), 6.85$ $(\mathrm{d}, J=8.3 \mathrm{~Hz}, 1 \mathrm{H}), 4.09(\mathrm{~s}, 3 \mathrm{H}) .{ }^{13} \mathrm{C} \mathrm{NMR}(100 \mathrm{MHz}$, $\left.\mathrm{CDCl}_{3}\right) \delta(\mathrm{ppm}) 163.5,150.0,148.8,147.9,139.6,131.8$, 129.5, 128.1, 117.7, 116.4, 111.7, 53.3. Elemental analysis: calcd (\%) for $\mathrm{C}_{12} \mathrm{H}_{9} \mathrm{~N}_{3} \mathrm{O}_{2}$ (227.22): C 63.43, H 3.99; found: C 63.68, H 4.13.

1-(5-(Benzo $[c][1,2,5]$ oxadiazol-4-yl)thiophen-2-

yl)ethan-1-one (19): Following the general procedure A using 1-(5-bromothiophen-2-yl)ethan-1-one (205 mg, 1 $\mathrm{mmol})$ and benzofurazan $(138 \mathrm{mg}, 1.15 \mathrm{mmol})$, the residue was purified by flash chromatography on silica gel (pentane-EtOAc, 80-20) to afford the desired compound 19 $(154 \mathrm{mg}, 63 \%)$ as a yellow solid $\left(\mathrm{MP}=205-207{ }^{\circ} \mathrm{C}\right) .{ }^{1} \mathrm{H}$ NMR $\left(400 \mathrm{MHz}, \mathrm{CDCl}_{3}\right) \delta(\mathrm{ppm}) 8.17(\mathrm{~d}, J=4.0 \mathrm{~Hz}, 1 \mathrm{H})$, $7.90-7.83(\mathrm{~m}, 1 \mathrm{H}), 7.78(\mathrm{~d}, J=4.0 \mathrm{~Hz}, 1 \mathrm{H}), 7.75(\mathrm{~d}, J=$ $6.8 \mathrm{~Hz}, 1 \mathrm{H}), 7.51(\mathrm{dd}, J=6.8,9.0 \mathrm{~Hz}, 1 \mathrm{H}), 2.64(\mathrm{~s}, 3 \mathrm{H})$. ${ }^{13} \mathrm{C} \mathrm{NMR}\left(100 \mathrm{MHz}, \mathrm{CDCl}_{3}\right) \delta(\mathrm{ppm}) 190.6,149.5,147.3$, $145.1,144.7$, 133.3, 131.6, 129.8, 127.6, 123.3, 116.3, 26.8. Elemental analysis: calcd $(\%)$ for $\mathrm{C}_{12} \mathrm{H}_{8} \mathrm{~N}_{2} \mathrm{O}_{2} \mathrm{~S}$ (244.26): C 59.01, H 3.30; found: C 58.87, H 3.11.

4-(5-Methoxybenzo[c][1,2,5]oxadiazol-4-

yl)benzonitrile (20): Following the general procedure $\mathbf{A}$ using 4-bromobenzonitrile (182 $\mathrm{mg}, 1 \mathrm{mmol})$ and 6methoxybenzofurazan (173 $\mathrm{mg}, 1.15 \mathrm{mmol})$, the residue was purified by flash chromatography on silica gel (pentane-Et $2 \mathrm{O}, 60-40$ ) to afford the desired compound $\mathbf{2 0}$ $(172 \mathrm{mg}, 68 \%)$ as an orange solid $\left(\mathrm{MP}=195-197^{\circ} \mathrm{C}\right) .{ }^{1} \mathrm{H}$ NMR (300 MHz, $\left.\mathrm{CD}_{2} \mathrm{Cl}_{2}\right) \delta(\mathrm{ppm}) 8.02-7.89(\mathrm{~m}, 3 \mathrm{H})$, $7.81(\mathrm{~d}, J=8.7 \mathrm{~Hz}, 2 \mathrm{H}), 7.58(\mathrm{~d}, J=9.8 \mathrm{~Hz}, 1 \mathrm{H}), 4.02(\mathrm{~s}$, $3 \mathrm{H}) .{ }^{13} \mathrm{C} \mathrm{NMR}\left(100 \mathrm{MHz}, \mathrm{CD}_{2} \mathrm{Cl}_{2}\right) \delta$ (ppm) 156.4, 150.0, $146.9,137.3,131.8,131.0,123.6,118.7,117.9,111.5$, 110.0, 57.6. Elemental analysis: calcd $(\%)$ for $\mathrm{C}_{14} \mathrm{H}_{9} \mathrm{~N}_{3} \mathrm{O}_{2}$ (251.25): C 66.93, H 3.61; found: C 67.05, H 3.88. 


\section{4-(3-Chlorophenyl)-5-}

methoxybenzo $[c][1,2,5]$ oxadiazole (21): Following the general procedure A using 1-bromo-3-chlorobenzene (191 $\mathrm{mg}, 1 \mathrm{mmol})$ and 6-methoxybenzofurazan $(173 \mathrm{mg}, 1.15$ $\mathrm{mmol})$, the residue was purified by flash chromatography on silica gel (pentane- $\mathrm{Et}_{2} \mathrm{O}, 90-10$ ) to afford the desired compound 21 (169 mg, 65\%) as a yellow solid (MP = 76$\left.79^{\circ} \mathrm{C}\right) .{ }^{1} \mathrm{H}$ NMR $\left(400 \mathrm{MHz}, \mathrm{CDCl}_{3}\right) \delta(\mathrm{ppm}) 7.89(\mathrm{~d}, J=$ $9.7 \mathrm{~Hz}, 1 \mathrm{H}), 7.79(\mathrm{~d}, J=1.5 \mathrm{~Hz}, 1 \mathrm{H}), 7.69(\mathrm{td}, J=1.5,7.5$ $\mathrm{Hz}, 1 \mathrm{H}), 7.51(\mathrm{~d}, J=9.7 \mathrm{~Hz}, 1 \mathrm{H}), 7.48-7.42(\mathrm{~m}, 1 \mathrm{H})$, $7.42-7.38(\mathrm{~m}, 1 \mathrm{H}), 3.98(\mathrm{~s}, 3 \mathrm{H}) .{ }^{13} \mathrm{C}$ NMR $(100 \mathrm{MHz}$, $\left.\mathrm{CDCl}_{3}\right) \delta(\mathrm{ppm}) 155.7,150.3,146.9,134.1,133.9,130.3$, 129.4, 128.5, 128.3, 123.9, 117.1, 111.3, 57.7. Elemental analysis: calcd (\%) for $\mathrm{C}_{13} \mathrm{H}_{9} \mathrm{ClN}_{2} \mathrm{O}_{2}$ (260.68): C 59.90, $\mathrm{H}$ 3.48; found: C 60.12 , H 3.27 .

\section{6-Methoxy-4-(2-}

nitrophenyl)benzo $[c][1,2,5]$ oxadiazole (22a): Following the general procedure A using 2-bromonitrobenzene (191 $\mathrm{mg}, 1 \mathrm{mmol}$ ) and 6-methoxybenzofurazan (173 mg, 1.15 $\mathrm{mmol})$, the residue was purified by flash chromatography on silica gel (pentane- $\mathrm{Et}_{2} \mathrm{O}, 60-40$ ) to afford the desired compound 22a (103 $\mathrm{mg}, 38 \%)$ as a yellow solid (MP = 200-202 $\left.{ }^{\circ} \mathrm{C}\right) .{ }^{1} \mathrm{H}$ NMR $\left(400 \mathrm{MHz}, \mathrm{CDCl}_{3}\right) \delta(\mathrm{ppm}) 8.20(\mathrm{~d}$, $J=8.1 \mathrm{~Hz}, 1 \mathrm{H}), 7.77(\mathrm{t}, J=7.6 \mathrm{~Hz}, 1 \mathrm{H}), 7.68(\mathrm{t}, J=7.9$ $\mathrm{Hz}, 1 \mathrm{H}), 7.59$ (d, $J=7.6 \mathrm{~Hz}, 1 \mathrm{H}), 7.10(\mathrm{~d}, J=2.0 \mathrm{~Hz}, 1 \mathrm{H})$, $6.92(\mathrm{~d}, J=2.0 \mathrm{~Hz}, 1 \mathrm{H}), 3.99(\mathrm{~s}, 3 \mathrm{H}) .{ }^{13} \mathrm{C}$ NMR $(100$ $\left.\mathrm{MHz}, \mathrm{CDCl}_{3}\right) \delta$ (ppm) 161.4, 150.1, 146.6, 133.5, 132.0, $130.3,130.2,128.8,127.2,125.2,89.6,56.2$. Elemental analysis: calcd (\%) for $\mathrm{C}_{13} \mathrm{H}_{9} \mathrm{~N}_{3} \mathrm{O}_{4}$ (271.23): $\mathrm{C} 57.57, \mathrm{H}$ 3.34; found: C 57.21, H 3.56. 22b ${ }^{1} \mathrm{H}$ NMR $(400 \mathrm{MHz}$, $\left.\mathrm{CDCl}_{3}\right) \delta(\mathrm{ppm}) 8.10(\mathrm{~d}, J=8.1 \mathrm{~Hz}, 1 \mathrm{H}), 7.95(\mathrm{~d}, J=9.7$ $\mathrm{Hz}, 1 \mathrm{H}), 7.75$ (t, $J=7.5 \mathrm{~Hz}, 1 \mathrm{H}), 7.70(\mathrm{~d}, J=7.5 \mathrm{~Hz}, 1 \mathrm{H})$, $7.61(\mathrm{t}, J=7.7 \mathrm{~Hz}, 1 \mathrm{H}), 7.46(\mathrm{~d}, J=9.7 \mathrm{~Hz}, 1 \mathrm{H}), 3.90$ (s, $3 \mathrm{H})$.

Procedure B (Palladium-catalyzed one-pot direct C4,C7-diarylation): To a $5 \mathrm{~mL}$ oven dried Schlenk tube, benzofurazan derivative ( $1 \mathrm{mmol})$, aryl bromide $(3 \mathrm{mmol})$, AcOK (294 mg, $3 \mathrm{mmol}$ ), DMA (4 mL) and $\mathrm{Pd}(\mathrm{OAc})_{2}(2.2$ $\mathrm{mg}, 0.01 \mathrm{mmol}, 2 \mathrm{~mol} \%$ ) were successively added. The reaction mixture was evacuated by vacuum-argon cycles ( 5 times) and stirred at $150{ }^{\circ} \mathrm{C}$ (oil bath temperature) for 16 hours. After cooling the reaction at room temperature and concentration, the crude mixture was purified by silica column chromatography to afford the desired diarylated products.

\section{4,7-Diphenylbenzo $[c][1,2,5]$ oxadiazole}

(23): Following the general procedure $\mathbf{B}$ using 1-bromobenzene (471 mg, $3 \mathrm{mmol}$ ) and benzofurazan (120 mg, $1 \mathrm{mmol})$, the residue was purified by flash chromatography on silica gel (pentane-Et $2 \mathrm{O}, 95-5)$ to afford the desired compound 23 (155 mg, 57\%) as a yellow solid (MP = 190-191 $\left.{ }^{\circ} \mathrm{C}\right) .{ }^{1} \mathrm{H}$ NMR (400 MHz, $\left.\mathrm{CDCl}_{3}\right) \delta(\mathrm{ppm}) 8.11-8.03(\mathrm{~m}, 4 \mathrm{H})$, $7.71(\mathrm{~s}, 2 \mathrm{H}), 7.62-7.54(\mathrm{~m}, 4 \mathrm{H}), 7.53-7.47(\mathrm{~m}, 2 \mathrm{H}) .{ }^{13} \mathrm{C}$ NMR (100 MHz, $\left.\mathrm{CDCl}_{3}\right) \delta(\mathrm{ppm})$ 149.3, 135.3, 129.2, $129.0,128.9,128.7,128.4$. Elemental analysis: calcd (\%) for $\mathrm{C}_{18} \mathrm{H}_{12} \mathrm{~N}_{2} \mathrm{O}$ (272.31): C 79.39, H 4.44; found: C 79.57, H 4.60 .

4,7-Bis(4-chlorophenyl)benzo[c][1,2,5]oxadiazole (24): Following the general procedure B using 1-bromo-4chlorobenzene $(574 \mathrm{mg}, 3 \mathrm{mmol})$ and benzofurazan (120 $\mathrm{mg}, 1 \mathrm{mmol}$ ), the residue was purified by flash chromatography on silica gel (pentane- $\mathrm{CH}_{2} \mathrm{Cl}_{2}, 80-20$ ) to afford the desired compound $\mathbf{2 4}(205 \mathrm{mg}, 60 \%)$ as a yellow solid $\left(\mathrm{MP}=230-232{ }^{\circ} \mathrm{C}\right) .{ }^{1} \mathrm{H}$ NMR $\left(400 \mathrm{MHz}, \mathrm{CDCl}_{3}\right) \delta$ (ppm) $8.02(\mathrm{~d}, J=8.3 \mathrm{~Hz}, 4 \mathrm{H}), 7.69$ (s, 2H), 7.55 (d, $J=$ $8.3 \mathrm{~Hz}, 4 \mathrm{H})$.

${ }^{13} \mathrm{C} \mathrm{NMR}\left(100 \mathrm{MHz}, \mathrm{CDCl}_{3}\right) \delta$ (ppm) 149.0, 135.4, 133.5, 129.6, 129.2, 128.5, 128.0. Elemental analysis: calcd (\%) for $\mathrm{C}_{18} \mathrm{H}_{10} \mathrm{Cl}_{2} \mathrm{~N}_{2} \mathrm{O}$ (341.19): C 63.37, $\mathrm{H}$ 2.95; found: $\mathrm{C}$ 63.18, H 3.09.

\section{4,7-Bis $(3,5-$}

bis(trifluoromethyl)phenyl)benzo $[c][1,2,5] \quad$ oxadiazole (25): Following the general procedure B using 1-bromo3,5-bis(trifluoromethyl)benzene $(879 \mathrm{mg}, 3 \mathrm{mmol})$ and benzofurazan $(120 \mathrm{mg}, 1 \mathrm{mmol})$, the residue was purified by flash chromatography on silica gel (pentane-toluene, 90-10) to afford the desired compound 25 (392 $\mathrm{mg}, 72 \%$ ) as a yellow solid $\left(\mathrm{MP}=156-159{ }^{\circ} \mathrm{C}\right) .{ }^{1} \mathrm{H}$ NMR $(300 \mathrm{MHz}$, $\left.\mathrm{CDCl}_{3}\right) \delta(\mathrm{ppm}) 8.53(\mathrm{~s}, 4 \mathrm{H}), 8.02(\mathrm{~s}, 2 \mathrm{H}), 7.86(\mathrm{~s}, 2 \mathrm{H})$ ${ }^{13} \mathrm{C}$ NMR $\left(100 \mathrm{MHz}, \mathrm{CDCl}_{3}\right) \delta(\mathrm{ppm}) 148.6,136.5,132.7$ $(\mathrm{q}, J=33.6 \mathrm{~Hz}), 129.6,128.4(\mathrm{~d}, J=2.6 \mathrm{~Hz}), 127.7,123.2$ (m), 123.1 (q, $J=270.1 \mathrm{~Hz})$. Elemental analysis: calcd (\%) for $\mathrm{C}_{22} \mathrm{H}_{8} \mathrm{~F}_{12} \mathrm{~N}_{2} \mathrm{O}$ (544.30): C 48.55, $\mathrm{H}$ 1.48; found: $\mathrm{C}$ 48.62, H 1.54 .

\section{4,7-Bis(6-(trifluoromethyl)pyridin-2-}

yl)benzo $[c][1,2,5]$ oxadiazole (26): Following the general procedure B using 2-bromo-6-(trifluoromethyl)pyridine (678 $\mathrm{mg}, 3 \mathrm{mmol})$ and benzofurazan $(120 \mathrm{mg}, 1 \mathrm{mmol}$ ), the residue was purified by flash chromatography on silica gel (pentane-toluene, 80-20) to afford the desired compound $26(263 \mathrm{mg}, 64 \%)$ as a yellow solid (MP $=209$ $\left.211{ }^{\circ} \mathrm{C}\right) .{ }^{1} \mathrm{H}$ NMR $\left(300 \mathrm{MHz}, \mathrm{CDCl}_{3}\right) \delta(\mathrm{ppm}) 8.96(\mathrm{~d}, J=$ $8.0 \mathrm{~Hz}, 2 \mathrm{H}), 8.84(\mathrm{~s}, 2 \mathrm{H}), 8.14(\mathrm{~d}, J=8.0 \mathrm{~Hz}, 2 \mathrm{H}), 7.78(\mathrm{~d}$, $J=8.0 \mathrm{~Hz}, 2 \mathrm{H}) .{ }^{13} \mathrm{C} \mathrm{NMR}\left(100 \mathrm{MHz}, \mathrm{CDCl}_{3}\right) \delta(\mathrm{ppm})$ $150.7,147.5,147.4$ (q, $J=34.9 \mathrm{~Hz}), 137.8,130.8,126.2$, 126.0, 120.3 (q, $J=275.7 \mathrm{~Hz}), 119.2$.Elemental analysis: calcd (\%) for $\mathrm{C}_{18} \mathrm{H}_{8} \mathrm{~F}_{6} \mathrm{~N}_{4} \mathrm{O}$ (410.28): C 52.70, H 1.97; found: C 52.89, H 1.63 .

Procedure C (acid-promoted the formation of quinoxalines): To a $5 \mathrm{~mL}$ oven dried Schlenk tube, arylated benzofurazan derivative $(0.5 \mathrm{mmol}), p$ toluenesulfonic acid ( $8.6 \mathrm{mg}, 0.05 \mathrm{mmol}), 2$-aminoethan-1ol $(0.45 \mathrm{~mL}, 7.5 \mathrm{mmol})$ were successively added. The reaction mixture wasstirred at $150{ }^{\circ} \mathrm{C}$ (oil bath temperature) for 72 hours. After cooling the reaction at room temperature and concentration, the crude mixture was purified by silica column chromatography to afford the desired arylated products.

5-(4-Chlorophenyl)quinoxaline (27): Following the general procedure $\mathbf{C}$ using 4-(4chlorophenyl)benzo[c][1,2,5]oxadiazole (6) (115 mg, 0.5 $\mathrm{mmol})$, the residue was purified by flash chromatography on silica gel (pentane- $\mathrm{CH}_{2} \mathrm{Cl}_{2}, 70-30$ ) to afford the desired compound $27(101 \mathrm{mg}, 84 \%)$ as a brown solid (MP $=109$ $\left.111{ }^{\circ} \mathrm{C}\right) .{ }^{1} \mathrm{H}$ NMR $\left(400 \mathrm{MHz}, \mathrm{CDCl}_{3}\right) \delta(\mathrm{ppm}) 8.97-8.85$ $(\mathrm{m}, 2 \mathrm{H}), 8.20(\mathrm{dd}, J=1.6,8.3 \mathrm{~Hz}, 1 \mathrm{H}), 7.88(\mathrm{t}, J=7.1 \mathrm{~Hz}$, $1 \mathrm{H}), 7.82(\mathrm{dd}, J=1.6,7.2 \mathrm{~Hz}, 1 \mathrm{H}), 7.63(\mathrm{~d}, J=8.5 \mathrm{~Hz}$ $2 \mathrm{H}), 7.51(\mathrm{~d}, J=8.5 \mathrm{~Hz}, 2 \mathrm{H}) .{ }^{13} \mathrm{C}$ NMR $(100 \mathrm{MHz}$, $\left.\mathrm{CDCl}_{3}\right) \delta(\mathrm{ppm}) 144.9,144.7,143.1,140.9,140.0,136.5$, $134.1,131.9,130.5,129.9,129.3,128.4$. Elemental analysis: calcd (\%) for $\mathrm{C}_{14} \mathrm{H}_{9} \mathrm{ClN}_{2}$ (240.69): $\mathrm{C}$ 69.86, $\mathrm{H}$ 3.77; found: C 69.90, H 4.02 .

5-(Naphthalen-1-yl)quinoxaline (28): Following the general procedure $\mathbf{C}$ using 4-(naphthalen-1yl)benzo[c][1,2,5] oxadiazole (15) $(123 \mathrm{mg}, 0.5 \mathrm{mmol})$, the residue was purified by flash chromatography on silica gel (pentane- $\mathrm{CH}_{2} \mathrm{Cl}_{2}, 70-30$ ) to afford the desired compound $28(111 \mathrm{mg}, 87 \%)$ as a brown solid $\left(\mathrm{MP}=100-103^{\circ} \mathrm{C}\right) .{ }^{1} \mathrm{H}$ NMR (400 MHz, $\left.\mathrm{CDCl}_{3}\right) \delta(\mathrm{ppm}) 8.88(\mathrm{~d}, J=1.8 \mathrm{~Hz}, 1 \mathrm{H})$, 
$8.77(\mathrm{~d}, J=1.8 \mathrm{~Hz}, 1 \mathrm{H}), 8.28(\mathrm{dd}, J=1.5,8.4 \mathrm{~Hz}, 1 \mathrm{H})$, $8.01-7.91(\mathrm{~m}, 3 \mathrm{H}), 7.86(\mathrm{dd}, J=1.5,7.1 \mathrm{~Hz}, 1 \mathrm{H}), 7.63$ $(\mathrm{dd}, J=7.0,8.2 \mathrm{~Hz}, 1 \mathrm{H}), 7.53(\mathrm{dd}, J=1.3,7.0 \mathrm{~Hz}, 1 \mathrm{H})$, 7.49 (ddd, $J=2.0,6.0,8.1 \mathrm{~Hz}, 1 \mathrm{H}), 7.38-7.30$ (m, 2H). ${ }^{13} \mathrm{C} \mathrm{NMR}\left(100 \mathrm{MHz}, \mathrm{CDCl}_{3}\right) \delta(\mathrm{ppm}) 144.9,142.9,142.3$, $140.5,136.5,133.5,132.7,132.0,129.7,129.4,128.5$, $128.3,128.1,126.3,125.9,125.7,125.2$. Elemental analysis: calcd (\%) for $\mathrm{C}_{18} \mathrm{H}_{12} \mathrm{~N}_{2}$ (256.31): C 84.35, H 4.72; found: C 84.14, H 4.96 .

5,8-Bis(3,5-bis(trifluoromethyl)phenyl)quinoxaline (29): Following the general procedure $\mathbf{C}$ using 4,7-bis(3,5bis(trifluoromethyl)phenyl)benzo[c] $[1,2,5]$ oxadiazole $(\mathbf{2 5})$ (272 $\mathrm{mg}, 0.5 \mathrm{mmol}$ ), the residue was purified by flash chromatography on silica gel (pentane- $\mathrm{CH}_{2} \mathrm{Cl}_{2}, 80-20$ ) to afford the desired compound $\mathbf{2 9}(227 \mathrm{mg}, 82 \%)$ as a brown solid $\left(\mathrm{MP}=200-203{ }^{\circ} \mathrm{C}\right) .{ }^{1} \mathrm{H}$ NMR $\left(400 \mathrm{MHz}, \mathrm{CDCl}_{3}\right) \delta$ (ppm) 8.98 (s, 2H), $8.30-8.16(\mathrm{~m}, 4 \mathrm{H}), 8.01(\mathrm{~s}, 2 \mathrm{H}), 8.00$ $(\mathrm{s}, 2 \mathrm{H}) .{ }^{13} \mathrm{C}$ NMR $\left(100 \mathrm{MHz}, \mathrm{CDCl}_{3}\right) \delta(\mathrm{ppm}) 145.3$, 140.7, 139.6, 138.9, 131.5 (q, $J=33.7 \mathrm{~Hz}), 130.8,130.3$, $123.4(\mathrm{q}, J=273.4 \mathrm{~Hz}), 121.8$. Elemental analysis: calcd

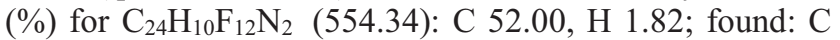
52.18, H 2.07 .

\section{Acknowledgements}

We thank the Algeria "Ministry of Higher Education and Scientific Research" for a fellowship to I.I. We thank CNRS and "Rennes Metropole" for providing financial support.

\section{References}

[1] Y. Li, Acc. Chem. Res. 2012, 45, 723-733.

[2] J. L. Rogers, L. Bayeh, T. H. Scheuermann, J. Longgood, J. Key, J. Naidoo, L. Melito, C. Shokri, D. E. Frantz, R. K. Bruick, K. H. Gardner, J. B. MacMillan, U. K. Tambar, J. Med. Chem. 2013, 56, 1739-1747.

[3]E. A. Chugunova, N. I. Akylbekov, A. D. Voloshina, N. V. Kulik, V. V. Zobov, V. M. Babaev, N. V. Gavrilov, A. R. Burilov, Synth. Commun. 2016, 46, 1560-1565.

[4] L. Wang, W. Huang, R. Li, D. Gehrig, P. W. M. Blom, K. Landfester, K. A. I. Zhang, Angew. Chem. Int. Ed. 2016, 55, 9783-9787.

[5] a) A. A. Vasil'ev, M. I. Struchkova, A. B. Sheremetev, F. S. Levinson, R. V. Varganov, K. A. Lyssenko, Russ. Chem. Bull. 2011, 60, 2306-2314; b) J. L. Rogers, L. Bayeh, T. H. Scheuermann, J. Longgood, J. Key, J. Naidoo, L. Melito, C. Shokri, D. E. Frantz, R. K. Bruick, K. H. Gardner, J. B. MacMillan, U. K. Tambar, J. Med. Chem. 2013, 56, 1739-1747; c) C. Song, Y. Ling, L. Jin, M. Zhang, D.-L. Chen, Y. He, Dalton Trans. 2016, 45, 190-197.

[6]a) H.-C. Ting, Y.-H. Chen, L.-Y. Lin, S.-H. Chou, Y.-H. Liu, H.-W. Lin, K.-T. Wong, ChemSusChem 2014, 7, 457-465; b) H.-C. Ting, Y.-T. Yang, C.-H. Chen, J.-H. Lee, J.-H. Chang, C.-I. Wu, T.-L. Chiu, C.-F. Lin, C.-L. Chung, K.-T. Wong, ChemSusChem 2016, 9, 14331441.

[7] J. Bouffard, T. M. Swager, Macromolecules 2008, 41, 5559-5562.
[8] H. Langhals, P. Knochel, A. Walter, S. Zimdars, Synthesis 2012, 44, 3465-3477.

[9] N. Nakamura, Y. Tajima, K. Sakai, Heterocycles 1982 , $17,235-245$.

[10] a) A. Ohta, Y. Akita, T. Ohkuwa, M. Chiba, R. Fukunaga, A. Miyafuji, T. Nakata, N. Tani, Y. Aoyagi, Heterocycles 1990, 31, 1951-1958; b) Y. Aoyagi, A. Inoue, I. Koizumi, R. Hashimoto, K. Tokunaga, K. Gohma, J. Komatsu, K. Sekine, A. Miyafuji, J. Kunoh, Heterocycles 1992, 33, 257-272.

[11] a) F. Kakiuchi, T. Kochi, Synthesis 2008, 3013-3039; b) L. Ackermann, R. Vicente, A. R. Kapdi, Angew. Chem. Int. Ed. 2009, 48, 9792-9826; c) F. Bellina, R. Rossi, Tetrahedron 2009, 65, 10269-10310; d) X. Chen, K. M. Engle, D.-H. Wang, J.-Q. Yu, Angew. Chem. Int. Ed. 2009, 48, 5094-5115; e) T. W. Lyons, M. S. Sanford, Chem. Rev. 2010, 110, 1147-1169; f) E. M. Beck, M. J. Gaunt, Top. Curr. Chem. 2010, 292, 85121 ; g) T. Satoh, M. Miura, Synthesis 2010, $3395-$ 3409; h) C.-L. Sun, B.-J. Li, Z.-J. Shi, Chem. Commun. 2010, 46, 677-685; i) S. H. Cho, J. Y. Kim, J. Kwak, S. Chang, Chem. Soc. Rev. 2011, 40, 5068-5083; j) N. Kuhl, M. N. Hopkinson, J. Wencel-Delord, F. Glorius, Angew. Chem. Int. Ed. 2012, 51, 10236-10254; k) B.-J. Li, Z.-J. Shi, Chem. Soc. Rev. 2012, 41, 5588-5598; 1) M. C. White, Synlett 2012, 23, 2746-2748; m) J. Yamaguchi, A. D. Yamaguchi, K. Itami, Angew. Chem. Int. Ed. 2012, 51, 8960-9009; n) S. I. Kozhushkov, L. Ackermann, Chem. Sci. 2013, 4, 886-896; o) R. Rossi, F. Bellina, M. Lessi, C. Manzini, Adv. Synth. Catal. 2014, 356, 17-117; p) M. Zhang, Y. Zhang, X. Jie, H. Zhao, G. Li, W. Su, Org. Chem. Front. 2014, 1, 843 895; q) M. R. Yadav, R. K. Rit, M. Shankar, A. K. Sahoo, Asian J. Org. Chem. 2015, 4, 846-864; r) K. Hirano, M. Miura, Chem. Lett. 2015, 44, 868-873; s) K. Yuan, J.-F. Soulé, H. Doucet, ACS Catal. 2015, 5, 978991; t) C. B. Bheeter, L. Chen, J.-F. Soulé, H. Doucet, Catal. Sci. Technol. 2016, 6, 2005-2049.

[12] J. F. Hartwig, M. A. Larsen, ACS Cent. Sci. 2016, 2, 281-292.

[13] a) J. Zhang, W. Chen, A. J. Rojas, E. V. Jucov, T. V. Timofeeva, T. C. Parker, S. Barlow, S. R. Marder, J. Am. Chem. Soc. 2013, 135, 16376-16379; b) J. Zhang, T. C. Parker, W. Chen, L. Williams, V. N. Khrustalev, E. V. Jucov, S. Barlow, T. V. Timofeeva, S. R. Marder, J. Org. Chem. 2016, 81, 360-370.

[14] F. Abdellaoui, C. Youssef, H. Ben Ammar, T. Roisnel, J.-F. Soulé, H. Doucet, ACS Catal. 2016, 6, 4248-4252.

[15] S. Chikhi, S. Djebbar, J.-F. Soulé, H. Doucet, Chem. Asian J. 2016, 11, 2443-2452.

[16] A. Jutand, M. Medio-Simons, B. Noverges Pedro, ChemCatChem 2017, DOI: 10.1002/cctc.201700041.

[17] V. A. Samsonov, Russ. Chem. Bull. 2007, 56, 25102512.

[18] a) O. S. Moustafa, J. Chin. Chem. Soc. 2003, 50, 1205-1208; b) G. Sakata, K. Makino, Y. Kurasawa, Heterocycles 1988, 27, 2481-2515; c) J. A. Pereira, A. M. Pessoa, M. N. D. Cordeiro, R. Fernandes, C. Prudêncio, J. P. Noronha, M. Vieira, Eur. J. Med. Chem. 2015, 97, 664-672. 


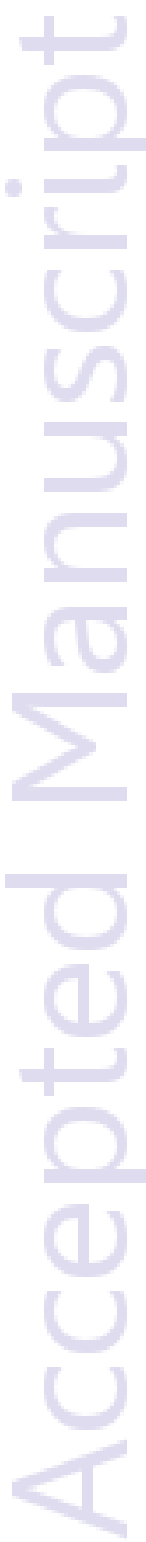




\section{FULL PAPER}

Palladium-Catalyzed Regioselective Direct Arylation of Benzofurazans at C4 Position

Adv. Synth. Catal. Year, Volume, Page - Page

I. Idris, F. Derridj, J.-F. Soulé,* H. Doucet*

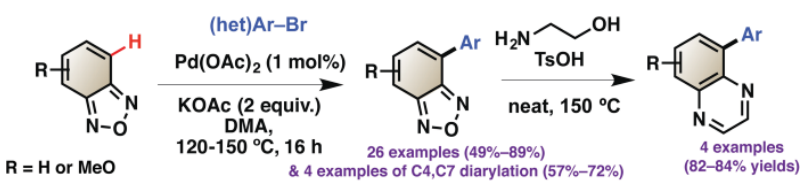

